\title{
Well-Posedness and Long Time Behavior for a Class of Fluid-Plate Interaction Models
}

\author{
Igor Chueshov and Iryna Ryzhkova \\ Department of Mathematics and Mechanics, \\ Kharkov National University, \\ 4 Svobody square, Kharkov, 61077, Ukraine \\ \{chueshov, ryzhkova\}@univer.kharkov.ua \\ http://mathphys.univer.kharkov.ua
}

\begin{abstract}
We deal with well-posedness and asymptotic dynamics of a class of coupled systems consisting of linearized 3D Navier-Stokes equations in a bounded domain and a classical (nonlinear) elastic plate/shell equation. We consider three models for plate/shell oscillations: (a) the model which accounts for transversal displacement of a flexible flat part of the boundary only, (b) the model for in-plane motions of a flexible flat part of the boundary, (c) the model which accounts for both transversal and longitudinal displacements. For all three cases we present wellposedness results and prove existence of a compact global attractor. In the first two cases the attractor is of finite dimension and possesses additional smoothness. We do not assume any kind of mechanical damping in the plate component in the case of models (a) and (b). Thus our results means that dissipation of the energy in the fluid due to viscosity is sufficient to stabilize the system in the latter cases.
\end{abstract}

Keywords: Fluid-structure interaction, nonlinear shell/plate, linearized 3D Navier-Stokes equations, global attractor, finite dimension.

\section{Introduction}

We consider a coupled (hybrid) system, which describes interaction of a homogeneous viscous incompressible fluid that occupies a bounded domain $\mathcal{O}$ with elastic plate/shell. Boundary $\partial \mathcal{O}$ of $\mathcal{O}$ consists of the (solid) walls of the container $S$ and a horizontal (flat) part $\Omega$, on which a thin (nonlinear) elastic shell or plate is placed. The motion of the fluid is described by linearized 3D NavierStokes equations. To describe deformations of the shell/plate we use several elastic models. In all cases we assume that large deflections of the shell produce small effect on the fluid. This corresponds to the case when the fluid fills the container which is large in comparison with the size of the shell/plate.

We note that the mathematical studies of the problem of interaction of viscous fluids and elastic plates/bodies have a long history. We refer to [31214] for the case of plates/membranes, to [10] in the case of moving elastic bodies, and to [1211] in the case of elastic bodies with fixed interface; see also the literature cited in these papers.

D. Hömberg and F. Tröltzsch (Eds.): CSMO 2011, IFIP AICT 391, pp. 328-337, 2013.

(C) IFIP International Federation for Information Processing 2013 


\section{Mathematical Description of the Models}

Fluid. Let $\mathcal{O} \subset \mathbb{R}^{3}$ be a bounded domain with sufficiently smooth boundary $\partial \mathcal{O}$. We assume that $\partial \mathcal{O}=\Omega \cup S$, where $\Omega \subset\left\{x=\left(x_{1} ; x_{2} ; 0\right): x^{\prime} \equiv\left(x_{1} ; x_{2}\right) \in \mathbb{R}^{2}\right\}$ with a smooth contour $\Gamma=\partial \Omega$ and $S$ is a surface lying in $\mathbb{R}_{-}^{3}=\left\{x_{3} \leq 0\right\}$. The exterior normal on $\partial \mathcal{O}$ is denoted by $n$. We have that $n=(0 ; 0 ; 1)$ on $\Omega$. The surface $S$ corresponds to solid walls of the container with a fluid, and $\Omega$ models an elastic shell or plate placed over the fluid.

To describe the fluid we consider the following linear Navier-Stokes equations in $\mathcal{O}$ for the fluid velocity field $v=v(x, t)=\left(v^{1}(x, t) ; v^{2}(x, t) ; v^{3}(x, t)\right)$ and the pressure $p(x, t)$ :

$$
\begin{gathered}
v_{t}-\nu \Delta v+\nabla p=G_{f} \quad \text { in } \mathcal{O} \times(0,+\infty), \\
\operatorname{div} v=0 \quad \text { in } \mathcal{O} \times(0,+\infty),
\end{gathered}
$$

where $\nu>0$ is the dynamical viscosity and $G_{f}$ is a volume force.

We denote by $T_{f}(v)$ the surface force exerted by the fluid on the shell which is equal to $\left.T n\right|_{\Omega}$, where $n$ is an outer unit normal to $\partial \mathcal{O}$ at $\Omega$ and $T=\left\{T_{i j}\right\}_{i, j=1}^{3}$ is the stress tensor of the fluid,

$$
T_{i j} \equiv T_{i j}(v)=\nu\left(v_{x_{j}}^{i}+v_{x_{i}}^{j}\right)-p \delta_{i j}, \quad i, j=1,2,3 .
$$

Since $n=(0 ; 0 ; 1)$ on $\Omega$, we have that

$$
T_{f}(v)=\left(\nu\left(v_{x_{3}}^{1}+v_{x_{1}}^{3}\right) ; \nu\left(v_{x_{3}}^{2}+v_{x_{2}}^{3}\right) ; 2 \nu v_{x_{3}}^{3}-p\right) .
$$

A specific form of this force as well as boundary conditions on $\Omega$ for the fluid depend on elastic plate/shell model we choose.

General Model (GM). We start with the full von Karman shallow shell model which accounts for both transversal and in-plane displacements (see [20|15|13|17] and the references therein). To this end we equip (11) and (2) with the (non-slip) boundary conditions imposed on the velocity field $v=v(x, t)$ :

$$
v=0 \quad \text { on } S, \quad v \equiv\left(v^{1} ; v^{2} ; v^{3}\right)=\left(u_{t}^{1} ; u_{t}^{2} ; w_{t}\right) \text { on } \Omega,
$$

where $u=u(x, t) \equiv\left(u^{1} ; u^{2} ; w\right)(x, t)$ is the displacement of the shell occupying $\Omega$. Here $w$ stands for the transversal displacement, $\bar{u}=\left(u^{1} ; u^{2}\right)$ - for the lateral (in-plane) displacements.

To describe the shell motion we use the full von Karman model which takes into account rotational inertia of the filaments and possible presence of in-plane acceleration terms (see the literature cited above):

$$
M_{\alpha}\left(w_{t t}+\gamma w_{t}\right)+\Delta^{2} w+\operatorname{trace}\{K \mathcal{N}(u)\}-\operatorname{div}\{\mathcal{N}(u) \nabla w\}=G_{3}-2 \nu v_{x_{3}}^{3}+p,
$$

and

$$
\varrho \bar{u}_{t t}=\operatorname{div}\{\mathcal{N}(u)\}+\left(G_{1}-\nu\left(v_{x_{3}}^{1}+v_{x_{1}}^{3}\right) ; G_{2}-\nu\left(v_{x_{3}}^{2}+v_{x_{2}}^{3}\right)\right),
$$


where $M_{\alpha}=1-\alpha \Delta, K=\operatorname{diag}\left(k_{1}, k_{2}\right)$, and

$$
\mathcal{N}(u) \equiv\left(\begin{array}{ll}
N_{11} & N_{12} \\
N_{12} & N_{22}
\end{array}\right)=\mathcal{C}\left(\epsilon_{0}(\bar{u})+w K+f(\nabla w)\right)
$$

with $\bar{u}=\left(u_{1} ; u_{2}\right), \mathcal{C}(\epsilon)=D[\mu \operatorname{trace} \epsilon \cdot I+(1-\mu) \epsilon]$, and

$$
\epsilon_{0}(\bar{u})=\frac{1}{2}\left(\nabla \bar{u}+\nabla^{T} \bar{u}\right), \quad f(s)=\frac{1}{2} s \otimes s, s \in \mathbb{R}^{2} .
$$

Here $D=E h /\left(1-\mu^{2}\right), E$ is Young's modulus, $0<\mu<1 / 2$ is Poisson's ratio, $h$ is the thickness of the shell, $\alpha>0$ and $\varrho \geq 0$ are constants taking into account rotational inertia and in-plane inertia of the shell, $\gamma \geq 0$ is a parameter which describes intensity of the viscous damping of the shell material. We denote by $G_{s h} \equiv\left(G_{1} ; G_{2} ; G_{3}\right)$ a (given) body force applied to the shell.

We impose the clamped boundary conditions on the shell:

$$
\left.u^{1}\right|_{\partial \Omega}=\left.u^{2}\right|_{\partial \Omega}=0
$$

and

$$
\left.w\right|_{\partial \Omega}=\left.\frac{\partial w}{\partial n}\right|_{\partial \Omega}=0 .
$$

We supply (10)-(77) with the initial data for the velocity field $v=\left(v^{1} ; v^{2} ; v^{3}\right)$ and the shell displacement vector $u=\left(u^{1} ; u^{2} ; w\right)$ of the form 1

$$
\left.v\right|_{t=0}=v_{0},\left.\quad u\right|_{t=0}=u_{0},\left.\quad w_{t}\right|_{t=0}=w_{1}, \varrho\left[\left.\bar{u}_{t}\right|_{t=0}-\bar{u}_{1}\right]=0,
$$

where $\bar{u}=\left(u^{1} ; u^{2}\right)$. Here $v_{0}=\left(v_{0}^{1} ; v_{0}^{2} ; v_{0}^{3}\right), u_{0}=\left(u_{0}^{1} ; u_{0}^{2} ; w_{0}\right), w_{1}$, and $\bar{u}_{1}=$ $\left(u_{1}^{1} ; u_{1}^{2}\right)$ are given vector functions which we will specify later.

From (2) and (3) we can also derive the compatibility condition

$$
\int_{\Omega} w\left(x^{\prime}, t\right) d x^{\prime}=\text { const for all } t \geq 0,
$$

which can be interpreted as preservation of the volume of the fluid.

Simplified Model 1 (SM1). This kind of models arises in the study of the problem of blood flows in large arteries (see, e.g., 12 and the references therein). The model assumes additional hypothesis that the transversal displacement $w$ of the plate is negligible relatively to the in-plane displacement $\left(u^{1} ; u^{2}\right)$. Thus we consider only longitudinal deformations of the plate and take into account only tangential shear forces which fluid exerts on the plate. Formally this means that we omit equation (4) and put $w \equiv 0$ in (3) and (5). Thus we arrive at the following boundary conditions imposed on the velocity field $v=v(x, t)$ :

$$
v=0 \quad \text { on } S, \quad v \equiv\left(v^{1} ; v^{2} ; v^{3}\right)=\left(u_{t} ; 0\right) \equiv\left(u_{t}^{1} ; u_{t}^{2} ; 0\right) \text { on } \Omega,
$$

\footnotetext{
${ }^{1}$ We put the multiplier $\varrho$ in the fourth relation of (8) to emphasize that this relation is not needed in the case of negligibly small in-plane inertia $(\varrho=0)$.
} 
where $u=u(x, t) \equiv\left(u^{1}(x, t) ; u^{2}(x, t)\right)$ is the in-plane displacement vector of the plate placed on $\Omega$ satisfying (5) with $\mathcal{N}_{0}(u)=\mathcal{C}\left(\epsilon_{0}(u)\right)$ instead of $\mathcal{N}$.

We assume that for this case the external (in-plane) force $\left(G^{1} ; G^{2}\right)$ in (5) is a nonlinear feedback force represented by a potential $\Phi$ :

$$
G^{i}=f^{i}\left(u^{1}, u^{2}\right) \equiv \frac{\partial \Phi\left(u^{1}, u^{2}\right)}{\partial u^{i}}, \quad i=1,2 .
$$

Since $v^{3}\left(x_{1} ; x_{2} ; 0\right)=0$ for $\left(x_{1} ; x_{2}\right) \in \Omega$ due to the second relation in (10), we have $v_{x_{i}}^{3}=0$ on $\Omega, i=1,2$. Thus after rescaling of the elastic constants we arrive at the following equations for the in-plane displacement $u=\left(u^{1} ; u^{2}\right)$ :

$$
u_{t t}^{i}-\Delta u^{i}-\lambda \partial_{x_{i}}[\operatorname{div} u]+\left.\nu v_{x_{3}}^{i}\right|_{x_{3}=0}+f^{i}(u)=0, \quad i=1,2,
$$

where $\lambda$ is a nonnegative parameter. We impose the clamped boundary conditions (6) for the displacement $u=\left(u^{1} ; u^{2}\right)$ on $\Gamma=\partial \Omega$. Thus we obtain

Problem (SM1): Find vector functions $v=\left(v^{1} ; v^{2} ; v^{3}\right)$ and $u=\left(u^{1} ; u^{2}\right)$ satis-

fying (in some sense) equations (11), (2), (10), (11), (6) and the initial data

$$
\left.v\right|_{t=0}=v_{0},\left.\quad u\right|_{t=0}=u_{0},\left.\quad u_{t}\right|_{t=0}=u_{1} .
$$

This problem with $\lambda=0$ and $f^{i}(u) \equiv 0$ was considered in [12] (see also the literature cited there) with the additional strong (Kelvin-Voight type) damping force applied to the interior of the plate. In contrast with [12] we do not assume the presence of mechanical damping terms in the plate component of the system and consider a nonlinearly forced model.

Simplified Model 2 (SM2). This model is concerned to dynamics of the transversal displacement $w$. The corresponding model assumes a special structure of the in-plane displacements $\bar{u}=\bar{u}(x, t) \equiv\left(u^{1}(x, t) ; u^{2}(x, t)\right)$ in (44) as a function of the transversal displacement $w$ only. Hence we neglect the equation in (5) (see, e.g., [1520] and the references therein). We also assume that $\alpha=0$. This formal procedure leads to the following boundary conditions imposed on the velocity field $v=v(x, t)$ :

$$
v=0 \text { on } S ; \quad v \equiv\left(v^{1} ; v^{2} ; v^{3}\right)=\left(0 ; 0 ; w_{t}\right) \text { on } \Omega .
$$

This and also (2) imply that $v_{x_{3}}^{3}=0$ and therefore the third (transversal) component $T_{f}(v)$ on $\partial \Omega$ is exactly the pressure $p$ of the fluid. Thus the transversal displacement $w=w(x, t)$ of the plate satisfies the following equation:

$$
w_{t t}+\Delta^{2} w+\mathcal{F}(w)=G_{p l}+\left.p\right|_{\Omega} \text { in } \Omega \times(0, \infty),
$$

where $G_{p l}$ is a given body force on the plate, $\mathcal{F}(u)$ is a nonlinear feedback force which will be specified later. As a result we obtain

Problem (SM2): Find the fluid velocity field $v=\left(v^{1} ; v^{2} ; v^{3}\right)$, the pressure $p$, and the transversal displacement of the plate $w$ satisfying (in some sense) equations (11), (2), (12), (13) and also compatibility condition (9), boundary conditions (7), and initial conditions of the form

$$
v(0)=v_{0}, \quad w(0)=w_{0}, \quad w_{t}(0)=w_{1} .
$$


Remark 1. We emphasize that even in the linear case due to the structure of the surface fluid forces $T_{f}(v)$ we cannot split system (11)-(8) into two sets of equations describing longitudinal and transversal plate movements separately, i.e., we cannot reduce the model in (GM) to the cases considered in the models in (SM1) and (SM2). The point is that in the case (SM1) equation (11) for longitudinal plate deformations does not contain the terms $v_{x_{i}}^{3}$ and the model does not require any compatibility conditions like (9) because the volume of the fluid obviously preserves. In the case of purely transversal displacements (see (SM2)) the force exerted on the plate by the fluid contains the pressure only. See also [9] for a further discussion.

Spaces and Notations. To describe fluid velocity fields we introduce the following spaces. Let $\mathcal{C}(\mathcal{O})$ be the class of $C^{\infty}$ vector-valued solenoidal (i.e., divergencefree) functions on $\overline{\mathcal{O}}$ which vanish in a neighborhood of $S$. We denote by $X$ the closure of $\mathcal{C}(\mathcal{O})$ with respect to the $L_{2}$-norm and by $V$ the closure of $\mathcal{C}(\mathcal{O})$ with respect to the $H^{1}(\mathcal{O})$-norm. One can see that

$$
\begin{gathered}
X=\left\{v=\left(v^{1} ; v^{2} ; v^{3}\right) \in\left[L_{2}(\mathcal{O})\right]^{3}: \operatorname{div} v=0, \gamma_{n} v \equiv(v, n)=0 \text { on } S\right\}, \\
V=\left\{v=\left(v^{1} ; v^{2} ; v^{3}\right) \in\left[H^{1}(\mathcal{O})\right]^{3}: \operatorname{div} v=0, v=0 \text { on } S\right\} .
\end{gathered}
$$

We refer to [19], for instance, for the details concerning spaces of this type.

To describe shell/plate displacements we use the Sobolev spaces $H^{s}(\Omega)$ and $H_{0}^{s}(\Omega)$. We also denote $\widehat{H}^{s}(\Omega)=H^{s}(\Omega) \cap \widehat{L}_{2}(\Omega)$ for $s \geq 0$, where $\widehat{L}_{2}(\Omega)$ is the subspace in $L_{2}(\Omega)$ consisting of functions with zero average over $\Omega$.

For $D=$ either $\mathcal{O}$ or $\Omega$ we denote by $\|\cdot\|_{D}$ the norm in $L_{2}(D)$ and by $\|\cdot\|_{s, D}$ the norm in $H^{s}(D)$ and keep the corresponding notations for the inner products.

\section{Results: Well-Posedness and Long-Time Dynamics}

General Model. We deal with weak (variational) solutions to (1)-(9) and consider the cases $\varrho>0$ and $\varrho=0$ simultaneously. This is possible due to an additional regularity estimate for the shell velocities, which follows from (3) and from the standard trace theorem. Even in the case $\varrho=0$ we have that

$$
\left\|w_{t}(t)\right\|_{H^{1 / 2}(\Omega)}^{2}+\left\|u_{t}^{1}(t)\right\|_{H^{1 / 2}(\Omega)}^{2}+\left\|u_{t}^{2}(t)\right\|_{H^{1 / 2}(\Omega)}^{2} \leq C\|\nabla v(t)\|_{\mathcal{O}}^{2}
$$

for every weak solution $(v(t) ; u(t))$. We use this observation to suggest unified way to prove a well-posedness result not distinguishing the cases $\varrho>0$ and $\varrho=0$ in contrast with [20] (see also [17]). We also note that in the case we neglect the inertia of longitudinal deformations $(\varrho=0)$ the equations in (5) become elliptic. However, we keep the initial data for the in-plane displacement $\left(u^{1} ; u^{2}\right)$. The point is that the first order evolution for $\left(u^{1} ; u^{2}\right)$ goes from the boundary condition for the fluid velocity in (3).

As a phase space we use

$$
\mathcal{H}=\left\{\begin{aligned}
\left\{\left(v_{0} ; u_{0} ; u_{1}\right) \in X \times W \times Y: v_{0}=u_{1} \text { on } \Omega\right\}, & \varrho>0, \\
\left\{\left(v_{0} ; u_{0} ; w_{1}\right) \in X \times W \times \widehat{H}_{0}^{1}(\Omega):\left(v_{0}\right)^{3}=w_{1} \text { on } \Omega\right\}, & \varrho=0,
\end{aligned}\right.
$$


where $w_{1}$ is the third component of the initial displacement velocity $u_{1}$ and

$$
W=H_{0}^{1}(\Omega) \times H_{0}^{1}(\Omega) \times \widehat{H}_{0}^{2}(\Omega), \quad Y=L_{2}(\Omega) \times L_{2}(\Omega) \times \widehat{H}_{0}^{1}(\Omega) .
$$

Our main result concerning (GM) is the following well-posedness theorem.

Theorem 1. Assume that $\alpha>0, \gamma \geq 0, \varrho \geq 0$, and

$$
U_{0} \in \mathcal{H}, \quad G_{f} \in V^{\prime}, \quad G_{s h} \in\left[H^{-1 / 2}(\Omega)\right]^{2} \times H^{-1}(\Omega)
$$

(in the case $\varrho=0$ the data $\bar{u}_{1}$ are not fixed). Then for any interval $[0, T]$ there exists a unique weak solution $(v(t) ; u(t))$ to (1) -(9) with the initial data $U_{0}$. This solution satisfies an energy balance equality and generates a continuous (both in strong and weak sense) evolution semigroup $S_{t}$ in the space $\mathcal{H}$. The evolution operator $S_{t}$ is defined as follows

- case $\varrho>0: S_{t}\left(v_{0} ; u_{0} ; u_{1}\right) \equiv U(t)=\left(v(t) ; u(t) ; u_{t}(t)\right)$, where the couple $(v(t) ; u(t))$ solves (1) - (9);

- case $\varrho=0: S_{t}\left(v_{0} ; u_{0} ; w_{1}\right) \equiv \bar{U}(t)=\left(v(t) ; u(t) ; w_{t}(t)\right)$, where $v(t)$ and $u(t)=$ $\left(u^{1}(t) ; u^{2}(t) ; w(t)\right)$ solves (11) - with $\varrho=0$.

Proof. We use the compactness method with Galerkin's approximations, which was inspired by the method developed in [3] for the case of a linear plate interacting with nonlinear Navier-Stokes equations. Uniqueness relies on the same idea as in [17] and involves Brésis-Gallouet type inequality. We follow the scheme of [13] in the proof of continuity properties of the solution and the energy equality. The details of the proof can be found in $[9$.

Remark 2. In the case $\alpha=0$ we can prove existence of weak solutions which satisfy an energy inequality using the same type of argument. Uniqueness of the solutions is still an open question. Sedenko's method does not work here because the nonlinearity is strongly supercritical when $\alpha=0$.

Our next result deals with global attractors. We recall (see, e.g., [518]) that global attractor of the dynamical system $\left(S_{t}, \mathcal{H}\right)$ is defined as a bounded closed set $\mathfrak{A} \subset \mathcal{H}$ which is invariant $\left(S_{t} \mathfrak{A}=\mathfrak{A}\right.$ for all $\left.t>0\right)$ and uniformly attracts all other bounded sets:

$$
\lim _{t \rightarrow \infty} \sup \left\{\operatorname{dist}_{\mathcal{H}}\left(S_{t} y, \mathfrak{A}\right): y \in B\right\}=0 \quad \text { for any bounded set } B \text { in } \mathcal{H} \text {. }
$$

Theorem 2. Assume that $\alpha>0, \gamma>0$, and the external forces satisfy

$$
G_{f} \equiv 0, \quad G_{s h}^{1}=G_{s h}^{2} \equiv 0, \quad \text { and } \quad G_{s h}^{3} \equiv g \in H^{-1}(\Omega)
$$

Let the set of the stationary points in $\mathcal{H}$ of the problem (11)-(9) is bounded. Then the corresponding evolution semigroup $S_{t}$ possesses a compact global attractor. 
To prove this theorem we apply J.Ball's method (in the form presented in [16]). To this end we need the property $\gamma>0$, i.e., assume a presence of mechanical damping in the transversal component of displacement. The question whether the system under consideration demonstrates compact long-time behavior without mechanical damping in the shell component is still open. The main obstacle in this case is that the dissipation of the energy in the fluid leads to the mechanical damping of order $1 / 2$ in the shell components (see (14)). This is not enough to stabilize kinetic energy of the plate, which is of the first order, (at least, uniformly). The same effect is valid for the model (SM2) with rotational inertia (see Remark 3 below). However, in the case of rotational inertia neglected $(\alpha=0)$ the matter differs. One can compare Remark 3 with the results for models (SM1) and (SM2), in which rotational inertia is not accounted for. We do not require any mechanical damping in these models and compact asymptotic dynamics of the corresponding systems is guaranteed by viscous dissipation in the fluid. Moreover, in these cases we can establish finite-dimensionality of the the corresponding attractors and also their smoothness. See [48] for details.

Simplified Model 1. We assume that the plate force potential $\Phi(u) \in C^{2}\left(\mathbb{R}^{2}\right)$ is a nonnegative polynomially bounded function,

$$
\left|\frac{\partial \Phi(u)}{\partial u^{i} \partial u^{j}}\right| \leq C\left(1+|u|^{p}\right), \quad i, j=1,2, \quad u=\left(u^{1} ; u^{2}\right) \in \mathbb{R}^{2},
$$

and the following dissipativity condition holds: for any $\delta>0$ there exist $c_{1}(\delta)>0$ and $c_{2}(\delta) \geq 0$ such that

$$
\sum_{i=1,2} u^{i} f^{i}(u)-c_{1}(\delta) \Phi(u)+\delta|u|^{2} \geq-c_{2}(\delta) \text { with } f^{i}(u)=\frac{\partial \Phi(u)}{\partial u^{i}} .
$$

We can consider as examples

$$
\Phi(u)=\psi_{0}\left(\left|u^{1}\right|^{2}+\left|u^{2}\right|^{2}\right) \text { or } \Phi(u)=\psi_{1}\left(u^{1}\right)+\psi_{2}\left(u^{2}\right),
$$

where $\psi_{i}(s)$ are nonnegative polynomials.

We use the following phase space for this model:

$$
\mathcal{H}=\{v \in X:(v, n)=0 \text { on } \Omega\} \times\left[H_{0}^{1}(\Omega)\right]^{2} \times\left[L_{2}(\Omega)\right]^{2} .
$$

Theorem 3 (Well-Posedness). Let $U_{0} \in \mathcal{H}$. Then for any interval $[0, T]$ there exists a unique weak solution $(v(t) ; u(t))$ to problem (SM1) for which an energy balance equality holds. Moreover, this solution defines a continuous evolution operator $S_{t}: \mathcal{H} \mapsto \mathcal{H}$ by the formula

$$
S_{t}\left(v_{0} ; u_{0} ; u_{1}\right)=\left(v(t) ; u(t) ; u_{t}(t)\right),
$$

where the couple $(v(t) ; u(t))$ solves problem (SM1), and there exists a constant $a_{R, T}>0$ such that for any couple of initial data possessing the property $\|U\|_{\mathcal{H}},\|\hat{U}\|_{\mathcal{H}} \leq R$ we have

$$
\left\|S_{t} U-S_{t} \hat{U}\right\|_{\mathcal{H}}^{2}+\int_{0}^{t}\|\nabla(v-\hat{v})\|_{\mathcal{O}}^{2} d \tau \leq a_{R, T}\|U-\hat{U}\|_{\mathcal{H}}^{2}, \quad \forall t \in[0, T],
$$


where $S_{t} U=\left(v(t) ; u(t) ; u_{t}(t)\right)$ and $S_{t} \hat{U}=\left(\hat{v}(t) ; \hat{u}(t) ; \hat{u}_{t}(t)\right)$. In the linear case $\Phi(u) \equiv 0$ problem (SM1) generates an exponentially stable $C_{0}$-semigroup of contractions $e^{-t \mathcal{A}}$ in $\mathcal{H}$ : there exist $C, \alpha>0$ such that

$$
\left\|e^{-t \mathcal{A}} U\right\|_{L(\mathcal{H}, \mathcal{H})} \leq C e^{-\alpha t} \text { for all } t>0
$$

We refer to 4 for the details. We note that property (16) improves the result in 12 which states the strong stability only.

The following result shows that model (SM1) demonstrates finite dimensional long-time dynamics.

Theorem 4 (Global Attractor). The dynamical system $\left(\mathcal{H}, S_{t}\right)$ generated by (SM1) possesses a compact global attractor $\mathfrak{A}$ of finite fractal dimension 2 . If relation (15) holds with $c_{2}(\delta) \equiv 0$, then the global attractor $\mathfrak{A}$ consists of a single point, $\mathfrak{A}=\{(0 ; 0 ; 0)\}$, which is exponentially attractive, i.e. there exist $c_{R}>0$ and $\alpha>0$ such that

$$
\left\|S_{t} U\right\|_{\mathcal{H}} \leq c_{R} e^{-\alpha t} \quad \text { for any } U \in \mathcal{H} \text { such that }\|U\|_{\mathcal{H}} \leq R
$$

It is well-known (see, e.g., 18]), that to prove the existence of a compact global attractor it is sufficient to show that the system is dissipative and asymptotically smooth. To prove dissipativity we use an appropriate Lyapunov function. As for asymptotic smoothness of the system and finite-dimensionality of the attractor, we rely on recently developed approach based on stabilizability estimates (see 67] and the references therein). We refer to [4] for further details.

Simplified Model 2. We impose the following hypotheses concerning the nonlinear feedback force $\mathcal{F}(u)$ in the plate equation (13).

(F1) $\mathcal{F}(u)$ is locally Lipschitz from $H_{0}^{2-\epsilon}(\Omega)$ into $H^{-1 / 2}(\Omega)$ for some $\epsilon>0$. (F2) There exists a $C^{1}$-functional $\Pi(u)$ on $H_{0}^{2}(\Omega)$ such that $\mathcal{F}(u)=\Pi^{\prime}(u)$.

(F3) The plate force potential $\Pi$ is bounded on bounded subsets of $H_{0}^{2}(\Omega)$, and there exist $\eta<1 / 2$ and $c \geq 0$ such that

$$
\eta\|\Delta w\|_{\Omega}^{2}+\Pi(w) \geq-c, \quad \eta\|\Delta w\|_{\Omega}^{2}+(w, \mathcal{F}(w))_{\Omega} \geq-c \quad \forall w \in H_{0}^{2}(\Omega) .
$$

We can consider Kirchhoff, von Karman, or Berger nonlinearities as examples of nonlinear feedback (elastic) force $\mathcal{F}(u)$, see 8 for the details.

We use

$$
\mathcal{H}=\left\{\left(v_{0} ; w_{0} ; w_{1}\right) \in X \times \widehat{H}_{0}^{2}(\Omega) \times \widehat{L}_{2}(\Omega): v_{0}^{3}=w_{1} \text { on } \Omega\right\}
$$

as a phase space and deal with weak (variational) solutions.

Theorem 5 (Well-Posedness). Assume that $U_{0}=\left(v_{0} ; w_{0} ; w_{1}\right) \in \mathcal{H}, G_{f} \in V^{\prime}$, and $G_{p l} \in H^{-1 / 2}(\Omega)$. Then for any interval $[0, T]$ there exists a unique weak

\footnotetext{
${ }^{2}$ For the definition and basic properties of the fractal dimension see, e.g., [18.
} 
solution $(v(t) ; w(t))$ to problem (SM2) with the initial data $U_{0}$. The solution possesses the property

$$
U(t) \equiv\left(v(t) ; w(t) ; w_{t}(t)\right) \in C(0, T ; \mathcal{H})
$$

and satisfies the energy balance equality

$$
\mathcal{E}\left(v(t), w(t), w_{t}(t)\right)+\nu \int_{0}^{t}\|\nabla v\|_{\mathcal{O}}^{2} d \tau=\mathcal{E}\left(v_{0}, w_{0}, w_{1}\right)+\int_{0}^{t}\left(G_{f}, v\right)_{\mathcal{O}} d \tau
$$

for every $t>0$, where the energy functional $\mathcal{E}$ is defined by the relation

$$
\mathcal{E}\left(v, w, w_{t}\right)=\frac{1}{2}\left(\|v\|_{\mathcal{O}}^{2}+\left\|w_{t}\right\|_{\Omega}^{2}+\|\Delta w\|_{\Omega}^{2}\right)+\int_{\Omega} \Pi(w(x)) d x-\left(G_{p l}, w\right)_{\Omega} .
$$

Moreover, there exists a constant $a_{R, T}>0$ such that for any couple of weak solutions $U(t)=\left(v(t) ; w(t) ; w_{t}(t)\right)$ and $\hat{U}(t)=\left(\hat{v}(t) ; \hat{w}(t) ; \hat{w}_{t}(t)\right)$ with the initial data possessing the property $\left\|U_{0}\right\|_{\mathcal{H}},\left\|\hat{U}_{0}\right\|_{\mathcal{H}} \leq R$ we have

$$
\|U(t)-\hat{U}(t)\|_{\mathcal{H}}^{2}+\int_{0}^{t}\|\nabla(v-\hat{v})\|_{\mathcal{O}}^{2} d \tau \leq a_{R, T}\left\|U_{0}-\hat{U}_{0}\right\|_{\mathcal{H}}^{2}, \quad t \in[0, T] .
$$

In the case $G_{f} \equiv 0, G_{p l} \equiv 0, \mathcal{F}(u) \equiv 0$ the problem generates a strongly continuous exponentially stable contraction semigroup $T_{t}$ on $\mathcal{H}$.

Using the same approach as in the proof of Theorem 4 we can establish the following result (see [8] for details).

Theorem 6 (Global Attractor). The dynamical system $\left(S_{t}, \mathcal{H}\right)$ generated by (SM2) possesses a compact global attractor $\mathfrak{A}$. Moreover,

(1) $\mathfrak{A}$ is the unstable set emanating from the set of equilibria $\mathcal{N}, \mathfrak{A}=\mathbb{M}_{+}(\mathcal{N})$;

(2) the attractor has finite fractal dimension;

(3) any trajectory $\gamma=\left\{\left(v(t) ; w(t) ; w_{t}(t)\right): t \in \mathbb{R}\right\}$ from the attractor $\mathfrak{A}$ possesses the property $\left(v_{t} ; w_{t} ; w_{t t}\right) \in L_{\infty}\left(\mathbb{R} ; X \times \widehat{H}_{0}^{2}(\Omega) \times \widehat{L}_{2}(\Omega)\right)$, and there is $R>0$ such that $\left(\left\|v_{t}\right\|_{\mathcal{O}}^{2}+\left\|w_{t}\right\|_{2, \Omega}^{2}+\left\|w_{t t}\right\|_{\Omega}^{2}\right) \leq R^{2}$ for all $t \in \mathbb{R}$ and $\gamma \subset \mathfrak{A}$.

We recall (see, e.g., [18]) that the unstable set $\mathbb{M}_{+}(\mathcal{N})$ emanating from some set $\mathcal{N} \subset \mathcal{H}$ is a subset of $\mathcal{H}$ such that for each $z \in \mathbb{M}_{+}(\mathcal{N})$ there exists a full trajectory $\{y(t): t \in \mathbb{R}\}$ satisfying $y(0)=z$ and $\operatorname{dist}(y(t), \mathcal{N}) \rightarrow 0$ as $t \rightarrow-\infty$.

Remark 3. We can consider model (SM2) with the rotational inertia accounted for (i. e., with the additional inertial term $-\alpha \Delta w_{t t}$ in the plate equation). In this case the phase space is $\left\{\left(v_{0} ; w_{0} ; w_{1}\right) \in \mathcal{H}: w_{1} \in H_{0}^{1}(\Omega)\right\}$ and the corresponding analog of Theorem 5 remains true, except the property of exponential stability of the linear semigroup $T_{t}$ (the case $G_{f} \equiv 0, G_{p l} \equiv 0, \mathcal{F}(u) \equiv 0$ ). To prove the existence of the attractor for (SM2) with the rotational inertia as in the case of (GM), we need to assume presence of rotational mechanical damping in the plate equation (see Theorem 2 and the comments after its statement). Whether model (SM2) with rotational inertia term and without mechanical dissipation demonstrates a compact long-time dynamics is still an open question. 


\section{References}

1. Avalos, G., Triggiani, R.: Semigroup well-posedness in the energy space of a parabolic hyperbolic coupled Stokes-Lamé PDE system of fluid-structure interaction. Discr. Contin. Dyn. Sys. Ser.S 2, 417-447 (2009)

2. Barbu, V., Grujić, Z., Lasiecka, I., Tuffaha, A.: Smoothness of weak solutions to a nonlinear fluid-structure interaction model. Indiana Univ. Math. J. 57, 1173-1207 (2008)

3. Chambolle, A., Desjardins, B., Esteban, M., Grandmont, C.: Existence of weak solutions for the unsteady interaction of a viscous fluid with an elastic plate. J. Math. Fluid Mech. 7, 368-404 (2005)

4. Chueshov, I.: A global attractor for a fluid-plate interaction model accounting only for longitudinal deformations of the plate. Math. Meth. Appl. Sci. 34, 1801-1812 (2011)

5. Chueshov, I.: Introduction to the Theory of Infinite-Dimensional Dissipative Systems. Acta, Kharkov (1999) (in Russian); English translation: Acta, Kharkov (2002); http://www.emis.de/monographs/Chueshov/

6. Chueshov, I., Lasiecka, I.: Long-Time Behavior of Second Order Evolution Equations with Nonlinear Damping. In: Chueshov, I., Lasiecka, I. (eds.) Memoirs of AMS, vol. 195(912). AMS, Providence (2008)

7. Chueshov, I., Lasiecka, I.: Von Karman Evolution Equations. Springer, New York (2010)

8. Chueshov, I., Ryzhkova, I.: A global attractor for a fluid-plate interaction model. Preprint ArXiv:1109.4324v1 (September 2011), to appear in Comm. Pure Appl. Anal.

9. Chueshov, I., Ryzhkova, I.: Unsteady interaction of a viscous fluid with an elastic shell modeled by full von Karman equations. Preprint ArXiv:1112.6094v1 (December 2011), To appear in J. Differ. Equat.

10. Coutand, D., Shkoller, S.: Motion of an elastic solid inside an incompressible viscous fluid. Arch. Ration. Mech. Anal. 176, 25-102 (2005)

11. Du, Q., Gunzburger, M.D., Hou, L.S., Lee, J.: Analysis of a linear fluid-structure interaction problem. Discrete Contin. Dyn. Syst. 9, 633-650 (2003)

12. Grobbelaar-Van Dalsen, M.: Strong stability for a fluid-structure model. Math. Methods Appl. Sci. 32, 1452-1466 (2009)

13. Koch, H., Lasiecka, I.: Hadamard well-posedness of weak solutions in nonlinear dynamic elasticity-full von Karman systems. In: Prog. Nonlinear Differ. Equ. Appl., vol. 50, pp. 197-216. Birkhäuser, Basel (2002)

14. Kopachevskii, N., Pashkova, Y.: Small oscillations of a viscous fluid in a vessel bounded by an elastic membrane. Russian J. Math. Phys. 5(4), 459-472 (1998)

15. Lagnese, J.: Boundary Stabilization of Thin Plates. SIAM, Philadelphia (1989)

16. Moise, I., Rosa, R., Wang, X.: Attractors for non-compact semigroups via energy equations. Nonlinearity 11, 1369-1393 (1998)

17. Sedenko, V.I.: On the uniqueness theorem for generalized solutions of initialboundary problems for the Marguerre-Vlasov vibrations of shallow shells with clamped boundary conditions. Appl. Math. Optim. 39, 309-326 (1999)

18. Temam, R.: Infinite-Dimensional Dynamical Dystems in Mechanics and Physics. Springer, New York (1988)

19. Temam, R.: Navier-Stokes Equations: Theory and Numerical Analysis, 1984th edn. AMS Chelsea Publishing, Providence (2001)

20. Vorovich, I.I.: On some direct methods in nonlinear oscillations of shallow shells. Izvestiya AN SSSR, Matematika 21(6), 142-150 (1957) (in Russian) 